\title{
An Experimental Performance Evaluation of Touchstone Delta Concurrent File System*
}

\author{
Rajesh R. Bordawekar Alok N. Choudhary Juan Miguel del Rosario ${ }^{\dagger}$ \\ NPAC and ECE Dept., 121 Link Hall, \\ Syracuse University, Syracuse, NY 13210
}

\begin{abstract}
For a high-performance parallel machine to be a scalable system, it must also have a scalable parallel $\mathrm{I} / \mathrm{O}$ system. This paper presents an experimental evaluation of the Intel Touchstone Delta's Concurrent File System (CFS). The main objective of the study is to determine the maximum file read/write rates for various configurations of $1 / O$ and compute nodes. In addition, we study the effects of file access modes, buffer sizes and file sizes on the system performance. In most cases, the result shows that performance of CFS scales as the number of disks is increased, but the sustained performance improvements are much lower than the system's peak capacity. We observe that the performance of CFS scales with the number of processors in the beginning, however, a plateu a quickly reached due to the $1 / 0$ system bottleneck and enormous software overhead, especially that of synchronization. Finally we also show that the performance of the CFS can greatly vary for various data distributions commonly employed in scientific and engineering applications.
\end{abstract}

\section{INTRODUCTION}

During the last decade, processor speeds have increased tremendously and this trend is likely to continue for the foreseeable future. During the same period, several important strides have been made in high-performance computing architectures. High speed processors coupled with massive parallelism are expected to provide computing power in teraflops in the next few years.

- This work was sponsored in part by DARPA under contract \# DABT63-91-C-0028 and NSF MIP-9110810. The content of the information does not necessarily reflect the position or the policy of the Government and no official endorsement should be inferred.

${ }^{\dagger}$ NPAC and CS Dept. Syracuse University

Permission to copy without fee all or part of this material is granted provided that the copies are not made or distributed for direct commercial advantage, the ACM copyright notice and the title of the publication and its date appear, and notice is given that copying is by permission of the Association for Computing Machinery. To copy otherwise, or to republish, requires a fee and/or specific permission.

ICS-7/93 Tokyo, Japan

- 1993 ACM 0-89791-600-X/93/0007/0367...\$1.50
Some of the commercially available parallel computers include Intel Paragon [4], nCUBE [8], CM-5 [12]. They are computational instruments of choice in the scientific community and can be found in one form or another in almost every major academic and research institution. Several prototypes are also being developed at various industrial and academic institutions. However, compared to the attention accorded to processors, interconnection networks and memories of these parallel systems, very little attention has been paid to the scalability and performance of the I/O system.

Providing raw processing speed and large memories without balancing $1 / O$ capabilities, however, is not sufficient to solve many real-world problems. A balanced memory hierarchy which can supply data to processors at the required speeds is critical to the success of high performance computing. Although semiconductor memories have become cheaper and faster, I/O systems' performance has not kept pace with the advances in processor and memory speeds. Very few parallel $\mathrm{I} / \mathrm{O}$ systems have been developed which can balance the processor speeds and the I/O bandwidth.

In this paper, we focus on the experimental evaluation of the Concurrent File System of the Intel Touchstone Delta. The goal is to study the effects of various workloads on the performance of a parallel $I / O$ system. Based on the experimental study, we identify various parameters that affect the system in a significant way. We believe that the study can help users to use the system in a better fashion as well as provide important input to the designers of future systems.

The rest of the paper is organized as follows. In section 2, we describe the parallel I/O model and the Intel Touchstone Delta system. Section 3 discusses the evaluation methodologies and lists some of the experiments presented in the paper. Section 4 presents performance evaluation results for various experiments. Array distribution results are presented in section 5. Finally, we conclude the paper in section 6 . 


\section{THE TOUCHSTONE DELTA SYSTEM}

The Touchstone Delta system was developed by the Intel Corporation as a part of the Touchstone program. The Intel Touchstone Delta system is a message passing multicomputer consisting of processing nodes that communicate across the two dimensional mesh interconnection network.

The system supports various types of processing nodes (numeric, mass storage, gateway and service). Numeric nodes form the computational core of the system. The Delta system uses Intel i860 processors as the core of computational nodes. In addition the Delta has 32 Intel 80386 processors as the core of the $I / O$ nodes. Each $1 / O$ node has 8 Megabytes memory that serves as I/O cache. There are other processor nodes such as service nodes and ethernet nodes. The Delta is arranged as a mesh of $16^{*} 32$ compute nodes and has $16 \mathrm{I} / \mathrm{O}$ node on each side (Figure 1).

\subsection{CONCURRENT FILE SYSTEM}

The Intel Touchstone Delta system consists of mesh connected compute nodes with attached set of $1 / O$ nodes. Each I/O node is connected to 2 disks, each with 1.4 Gigabytes of space. I/O nodes do not run any application processes but provide disk services for all users. A file is uniformly distributed over 64 disks by default in a round-robin manner [10]. The stripe unit is $4 \mathrm{Kil}-$ bytes (one block). When a file is opened for reading or writing, data is accessed by default from 64 disks. A user, however, can restrict the number of disks on which a fle is distributed. All read-write transactions are carried out in an integral number of blocks, where each block size is 4 Kilobytes.

\subsection{CFS FILE STRUCTURE}

The CFS provides a UNIX view of a file to the application programs. Each CFS file has a header and a body. CFS file header stores file information such as file size, permission and link count. The file header is alway's allocated the first file block. In case of small files the header contains the data whereas for large files the header contains the pointers to the indirect blocks that store the data. When the file is striped across the disks, the file header is stored on the first disk and all the subsequent blocks are distributed in a round-robin fashion over the disks.

\subsection{THE I/O MODES}

Four 1/O modes are supported in the CFS. These are described below.

- Mode 0: In this mode, each node process has its own file pointer. This mode is specifically useful for large files to be shared among the nodes.
Here, sharing implies that the same data is accessed by nodes (replicated). This should be distinguished from sharing a file but distributing the data, i.e, when different nodes access different (and distinct) parts of a file. This mode is useful for accessing the file in GSA access pattern.

- Mode 1: In this mode, the compute nodes share a common file pointer. $I / O$ requests are serviced on a first-come-first-serve basis. Nodes can read and write at any point, but they use the same file pointer. Thus GSP file access pattern is obtained.

- Mode 2 : Mode 2 treats reads and writes as global operations. The set of compute nodes that open a concurrent file must read the file in a specified order (in the increasing order of the nodenumbers). This mode performs global synchronization in the sense that the second request by any node is blocked until the first request by all nodes in the set is complete. This mode supports synchronized common file pointer. Using this mode, nodes can perform variable length readwrite operations. Hence, the requests are serviced in a predefined order (increasing order of the node-numbers).

- Mode 3: Mode 3 is a synchronized ordered mode. The difference between mode 2 and mode 3 is that in mode 3 , all read/write operations must be of the same size. This mode also supports global synchronization. Hence, the requests can be serviced in any order, but still the second request by a node is blocked until the first request of all nodes is completed. Hence this mode can be used for obtaining GSI form of file access.

The data accessed by each processor depends upon the mode. During the write operation, the resultant size of the file created depends on the file mode used. Many scientific applications involve automatic distribution of the data across processors. Using different $\mathrm{I} / \mathrm{O}$ modes, it is very easy to decompose the data across the disks. Note the distinction between mode 0 and the other 3 modes. In mode 0 , reads/writes are to the same data in a shared file, whereas in other modes reads/writes are to distinct data (for each node) in the shared file.

\subsection{THE I/O NETWORK}

Touchstone Delta does not provide an independent $\mathrm{I} / \mathrm{O}$ network. The compute and I/O nodes share a common interconnection network. The same network is used for both interprocess communication and $1 / O$ communication.

In Touchstone Delta, for both interprocess communication and $1 / O$, messages travel in the form of packets. Touchstone Delta uses packet switched wormhole routing as a communication protocol $[5,6,11]$. Each node 


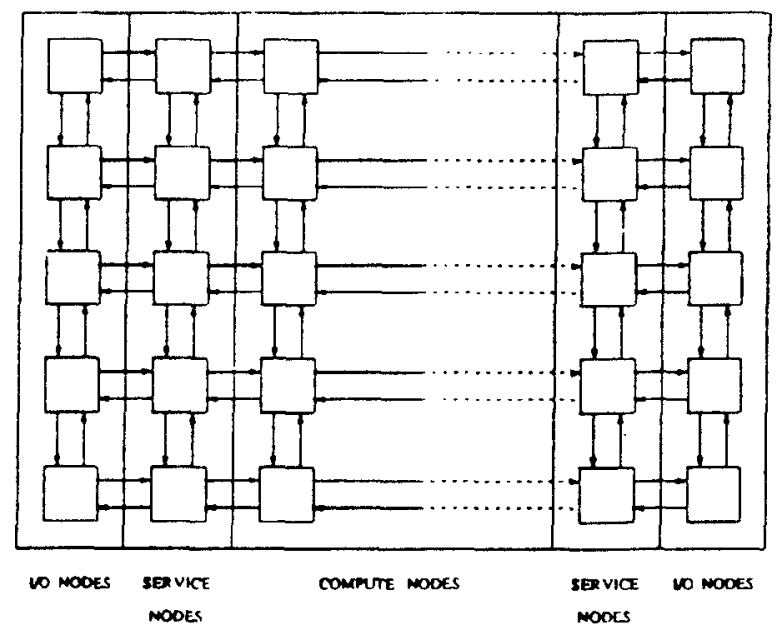

Figure 1: Intel Touchstone Delta System

of the machine is connected to the mesh using a mesh routine chip (MRC). The message travels from MRC to MRC unti it reaches the destination node.

Each message is split into packets of a fixed size (512 Bytes). On the physical level, the packet travels through the network in form of flits or flow control digits $[6,7]$. The packet follows a $X Y$ routing protocol. The $X Y$ direction is specified in the message header. In Touchstone Delta, the message packets always travel first along $X$ direction, and when it reaches the right column, the packet travels in the $Y$ direction.

Using the same network for interprocessor communication and I/O may cause serious network contention. Also since the $I / O$ nodes are physically at the edge of the mesh, the position of the compute nodes might affect the $1 / O$ performance. We will investigate these points in the experimental analysis of Touchstone Delta.

\section{CFS EVALUATION METHODOLOGY}

The overall performance of accessing data in a CFS depends on several factors including the number of compute nodes participating in an 1/O operation, size of access (buffer size), number of disks, block size, I/O mode and the overall available bandwidth from the $1 / 0$ system as well as that of the interconnection network (Figure 1). In principle, it is difficult to decouple the influence of some parameters on the performance. Our study includes the following experiments:

\subsection{SINGLE COMPUTE NODE}

- Single compute node and paged $1 / 0$
In paged $1 / O$ experiments, we study the effects of buffer size, node position, number of disks and the file size on the throughput. These experiments are carried out for smaller buffer sizes and relatively small file sizes.

- Single compute node and burst $I / O$

For burst $1 / 0$ experiments, the buffer size is very large. The factors that affect the burst mode throughput include the buffer size and the number of disks.

\subsection{MULTIPLE COMPUTE NODES}

- Multiple compute nodes and paged $1 / 0$

Multiple processors access the file system using various access modes. During multinode accesses, the CFS performance not only depends on the buffer size, number of processors and disks, but also on the file access modes. Hence, the paged I/O experiments are carried out for all the four file access modes.

- Multiple compute nodes and burst $I / O$

In burst $\mathrm{I} / \mathrm{O}$ mode, the buffer size is quite large compared to that in the paged mode. For such file accesses, the parameters that affect the throughput include the number of processors and the number of disks. Furthermore, we study the performance of the CFS when data is distributed using common data distributions found in typical scientific programs. 
Table 1: Definitions of various terms used in the chapter

\begin{tabular}{|c|c|}
\hline Term & Definition \\
\hline I. & The size of the file distributed over the disks. \\
\hline$V_{n}$ & The number of processors accessing the file. \\
\hline$F_{p}$ & $\begin{array}{l}\text { The amount of file data per processor. } \\
\qquad F_{p}=\frac{F}{N_{p}} \text {. } \\
\text { (True only for modes } 1,2 \text { and } 3 . \text { ) }\end{array}$ \\
\hline$T_{10}$ & The number of $\mathrm{I} / \mathrm{O}$ requests per processor. \\
\hline b.o & $\begin{array}{l}\text { The size of the data buffer. } \\
\text { Access size requested by the processor }\end{array}$ \\
\hline$x_{D}$ & Total number of disks (for Delta $N_{D}$ is 64 ). \\
\hline$B n$ & I Block Size ( A mount of data per block on each disk \\
\hline
\end{tabular}

\subsection{EFFECT OF INTERCONNECTION NETWORK ON I/O PERFORMANCE}

We will investigate the effects of using the common network for both interprocess communication and $\mathrm{I} / \mathrm{O}$. We will especially study the effect of compute node position on the $1 / O$ performance. The results will help in analyzing the mesh network performance.

Table 1 presents some definitions that will be used throughout this thesis.

\section{CFS PERFORMANCE EVALUATION}

The main objective of the CFS performance evaluation is to determine the maximum read-write rates observed for different configurations. Therefore, the experiments try to saturate the $I / O$ system with the $I / O$ requests so as to obtain a peak performance. Similar performance measurements have been used in the study of Intel iPSC $1 / O$ system $[3,9]$.

\subsection{SINGLE COMPUTE NODE}

The first part of the study aims to determine the maxinum $1 / O$ rates obtained for a single compute node. These studies are performed both for paged as well as burst I/O modes. Paged I/O performance is important for implementing and supporting node virtual memory to fetch or store pages on disks. Burst-mode $I / O$ is important for file accesses when a node requires reading/writing large files containing data for an application. For both types of workload we study the maximum throughput obtained from the I/O systems.

\section{PAGED $1 / 0$}

Since there is no virtual memory support currently available on the Delta system, virtual memory was simulated by opening a file and reading (writing) it using fixed size buffers. The buffer size $B_{\text {to }}$ indicates the amount of data fetched in each $I / O$ request. In each experiment, the compute node opens a file and reads (writes) it using fixed size buffers. Other parameters varied for the following experiments include the file size,

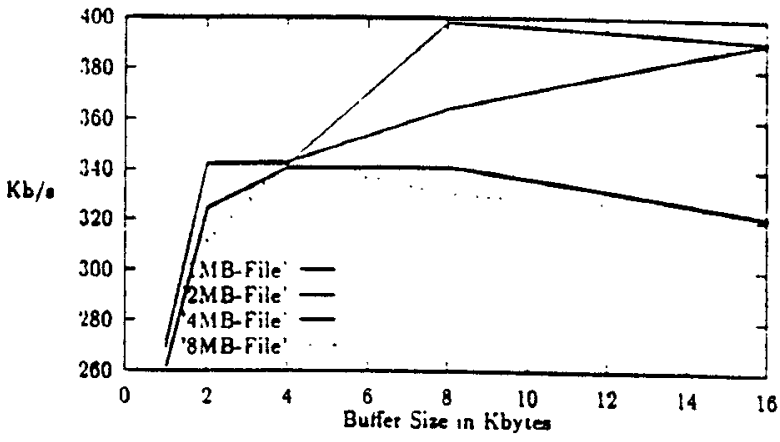

Figure 2: Single Compute Node - 32 I/O Nodes(64 Disks): Read Rates in $\mathrm{KB} / \mathrm{sec}$

buffer size, node position and the number of disks $V_{L}$. Figure 2 illustrates the performance of implementing paged $\mathrm{I} / \mathrm{O}$ using various buffer sizes.

As the buffer size increases from $1 \mathrm{~K}, N_{10}$ reduces and consequently the throughput increases. For a buffer size of about $4 \mathrm{k}$, the read rate is about $340 \mathrm{~K}$ bytes/sec for all file sizes. This convergence of performance occurs because the buffer size and the block size are both $4 \mathrm{Kbytes}$, and therefore, the requested size is same as the size of data read in one operation from the disk. Thus each $1 / O$ request for $4 \mathrm{~K}$ buffer results in reading the prefetched blocks from the node cache. Beyond $4 \mathrm{~K}$ buffer size, the throughput increases for small files as a function of buffer size whereas it degrades slightly for larger files. In summary, $4 \mathrm{~K}$ buffer size seems optimal for most of the files but for very small files, larger buffer sizes perform well. It should be noted that the throughput is not limited by the I/O system, but is limited by how fast a node request is generated. For the paged $1 / O$ experiments, new $I / O$ request is generated only when the previous one is completed. Similar results were obtained for a write operation.

In order to determine whether the position of a compute node in the network has any effect on read and write operations, different nodes at various locations in the mesh were chosen as shown in Table 2. Three were on one side of the mesh (Node Nos. $56,11,24$ ), two in middle of the mesh (Node Nos. 319,268 ), and the remaining three on the other side of the mesh. Keeping the buffer size fixed at $4 \mathrm{~K}$, file read times were observed for file sizes varying from $1 \mathrm{M}$ to $8 \mathrm{M}$. The files were distributed over 64 disks, so each side had an equal amount of data distributed on the nearby disks (32). The read times do not change significantly as the position of the node varies. This experiment shows that the distance that a request travels in the network does not have any significant impact on the performance when there is very little contention in the network. This also shows that the inter-node "hop times" between the nodes are negligible. 
Table 2: File Read Time is (ms)-Single compute node

\begin{tabular}{|c||c||c||c|c|}
\hline Sode Io. & $1 \mathrm{MB}$ & $2 \mathrm{MB}$ & $4 \mathrm{MB}$ & $8 \mathrm{MB}$ \\
\hline 56 & 2954 & 5836 & 11642 & 23230 \\
\hline 11 & 2926 & 5896 & 11754 & 23195 \\
\hline 24 & 3018 & 5836 & 11719 & 23314 \\
\hline 319 & 2968 & 5843 & 11694 & 23205 \\
\hline 205 & 2951 & 5936 & 11725 & 23215 \\
\hline 567 & 2994 & 5903 & 11941 & 23264 \\
\hline 535 & 3078 & 6096 & 11790 & 23460 \\
\hline 517 & 2972 & 5961 & 11897 & 23383 \\
\hline
\end{tabular}

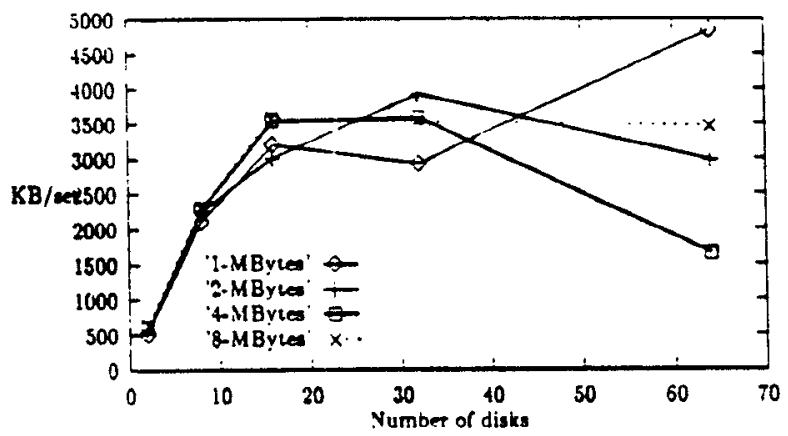

Figure 3: Read Rates for Single Compute Node-Burst Ifode

\section{BURST MODE I/O}

When the compute nodes require to read (write) a large amount of data (large fraction or an entire file) then the operation may be performed in a burst mode. In the burst mode. the buffer size is very large, maximum being the file size. Figures 3 and 4 show that burst mode operation is much faster than paged mode. Using 64 disks. IMB file was read in $203 \mathrm{~ms}$ giving a peak rate of $4.83 \mathrm{MBytes} / \mathrm{sec}$. The peak write rate was 1.39 $\mathrm{MB} / \mathrm{sec}$. The peak read rate obtained for paged mode was about $400 \mathrm{~KB} / \mathrm{sec}$. This increase in the throughput, compared to that in paged mode is observed due to the large number of $1 / O$ requests in paged $I / O$ mode where each request must be sent explicitly.

Generally for the single processor configuration, both for the paged and for the burst mode $\mathrm{I} / \mathrm{O}$, the read rates are much higher than the write rates. Also as the number of disk volumes increases the throughput increases upto a threshold. For large files, the trend will be the same as the " 8 -Mbytes" case shown in Figure 4. For small size files, the data is unevenly distributed on disks resulting in an imbalance, and therefore, we observe different trends.

\subsection{MULTIPLE COMPUTE NODES}

The most important use of a parallel $1 / O$ system and CFS is concurrent accesses by multiple processors. This section presents performance of the file system by vary-

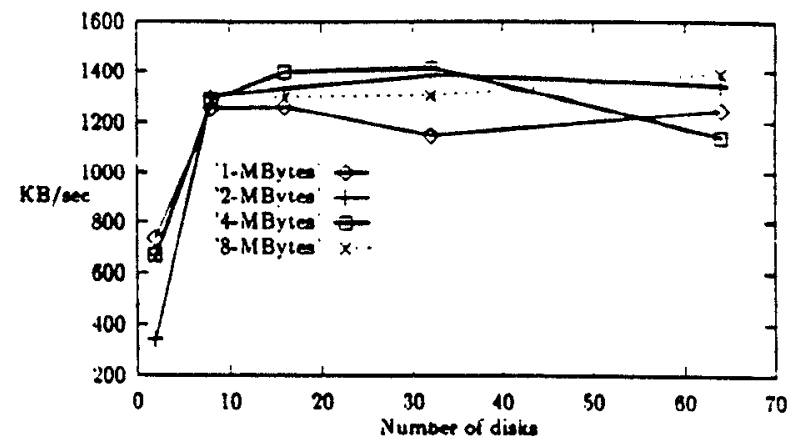

Figure 4: Write Rates for Single Compute Node-Burst Mode

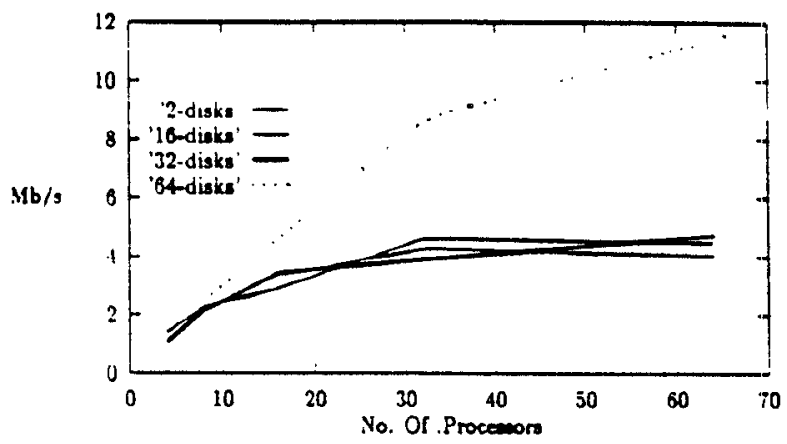

Figure 5: Read Rates For Multiple Compute Nodes Mode 0

ing different parameters such as the number of disks, the access modes and the number of processors.

\section{MODE O: PAGED I/O}

Mode 0 is useful for accessing shared files by multiple compute nodes. Each processor has its own file pointer. Note that write operations are not protected in the sense that the processors can overwrite each others data.

Figure 5 shows the read throughput for paged $1 / 0$ as a function of the number of processors for various number of disks. Figure 5 shows that there exists a threshold in terms of the number of disks beyond which a substantial performance gains can be expected. As the number of processors is increased, the performance does not change significantly when $N_{d}$ is increased from 2 to 32 disks. However for 64 disks, there is a significant jump in the performance. The throughput obtained for 64 processors is $11.2 \mathrm{MBytes} / \mathrm{sec}$. Whereas for 2 disks, the throughput is about $4.5 \mathrm{Mbytes} / \mathrm{sec}$. This shows that the "declustering" of the file data is very effective.

The throughput increases as the number of processors increases. Since each processor reads the same data, as the number of processors increases, the total amount of shared data accessed increases. However, the total time required to read the data increases slowly because 
Tiable 3: Multinode $\left(4^{*} 4\right)$ Burst Mode Thrcughput $(\mathrm{MB} / \mathrm{s})$ as a Function of Disk Volumes (Mode 0)

\begin{tabular}{|c|c|c|c|c|}
\hline File size & Butfer size & Disks & Read & Vrite \\
\hline 1.11 & 1.11 & 2 & 0.9329 & 0.748 \\
\hline 111 & 1.1 & 16 & 5.88 & 3.069 \\
\hline 1.11 & 111 & 32 & 11.11 & 3.147 \\
\hline 1.11 & 111 & 64 & 11.21 & 3.506 \\
\hline $4 \pi$ & 411 & 2 & 0.74 & 0.603 \\
\hline 4.11 & 4.11 & 16 & 3.630 & 2.0878 \\
\hline $4 N$ & $4 \mathrm{NI}$ & 32 & 7.705 & 4.947 \\
\hline 4.11 & 411 & 64 & 12.11 & 6.055 \\
\hline
\end{tabular}

one node's read acts as a prefetch command for others. Dire to the hardware constraints, we were not able to carry out the experiment for large number of processors (greater than 64).

Another interestirg point to observe is that for a small number of processors, the effect of the number of disks on the performance is negligible. That is, the performance is limited by the bandwidth available at the computational node side rather than at the I/O subsystem side.

\section{MODE O: BURST I/O}

Table 3 shows the performance of burst-mode read/write throughput as a function of number of disks (processor size $=16$ ). The specified buffer size at the application level is equal to the size of the file to be $\mathrm{read} / \mathrm{written}$. As we can observe. the performance improves as the number of disks is increased. For a given processor grid size, the performance depends on two parameters, namely, the file size and the number of disks. For smaller files (1 Mbytes/node), the performance saturates for smaller number of disks (32 disks) and beyond which the improvements diminish. However, as the file size increases, the threshold number of disks for which performance improves also increases as seen for the case of 4 MByte read/write per node.

Tables 4 and 5 presents the performance of burst mode $\mathrm{I} / \mathrm{O}$ when the number of computational nodes is varied from 16 to $512\left(N_{D}=64\right)$. In general for burst $1 / O$, good performance is obtained and the saturation occurs due to bandwidth limitation of the I/O subsystem. Reads perform better than writes. This is because reads can be performed from the $1 / O$ cache if a desired block exists in the cache due to an access by some other processor. However, all writes must be written onto the disks. The difference between paged and burst I/O performance is not significant because the system bandwidth is not limited.

\section{MODES 1. 2 AND 3 : PAGED 1/O}

These modes are useful for accessing data when the data set is distributed over multiple nodes.

The first experiment was used to observe the effect of different modes and the number of disks. In this
Table 4: Throughput $(\mathrm{MB} / \mathrm{s})$ of accessing $1 \mathrm{MB}$ file in Mode 0 (Burst Mode,64 Disks)

\begin{tabular}{|c||c|c|}
\hline Mesh Size & Write Rate (MB/sec) & Read Rate( $\ 1 \mathrm{~B} / \mathrm{sec})$ \\
\hline $4^{*} 4$ & 3.506 & 11.21 \\
\hline $4^{*} 8$ & 5.421 & 11.75 \\
\hline $8^{*} 8$ & 7.027 & 8.853 \\
\hline $8^{*} 16$ & 3.314 & 11.766 \\
\hline $16^{*} 16$ & 3.244 & 5.2819 \\
\hline $16^{*} 32$ & 2.839 & 11.992 \\
\hline
\end{tabular}

Table 5: Throughput $(\mathrm{MB} / \mathrm{s})$ of accessing $2 \mathrm{MB}$ fle in mode 0 (Burst Mode)

\begin{tabular}{|c||c||c|}
\hline Mesh Size & Write Rate(MB/sec) & Read Rate $(M \mathrm{BB} / \mathrm{sec})$ \\
\hline $4^{*} 4$ & 4.37 & 10.51 \\
\hline $4^{*} 8$ & 3.89 & 11.73 \\
\hline $16^{*} 16$ & 2.860 & 12.103 \\
\hline $16^{*} 32$ & 3.678 & 23.83 \\
\hline
\end{tabular}

experiment a data file of size 16 Mbytes was read using modes 1, 2 and 3 . As Figure 6 shows, for a $4^{*} 4$ processor grid the maximum throughput is obtained for mode 3 and with 64 disks. The peak rate in this case is $5.5 \mathrm{Mbytes} / \mathrm{sec}$. For mode 1, the peak speed is 5.12 Mbytes/sec. The lowest file read throughput is observed for mode 2.

Another important point to be noted is that as the number of disks decreases, the read throughput decreases. As the number of disks is decreased below a threshold (in this case 16 disks), the read rate reduces drastically. Hence, the optimal operating point in terms of cost-performance (no. of disks versus the throughput) will be near the knee of the curve.

In the next experiment, we use 64 disks (maximum available) and vary the number of processors. For this experiment, a $16 \mathrm{M}$ file was read using a $4 \mathrm{~K}$ buffer.

Figure 7 shows that as the number of processors

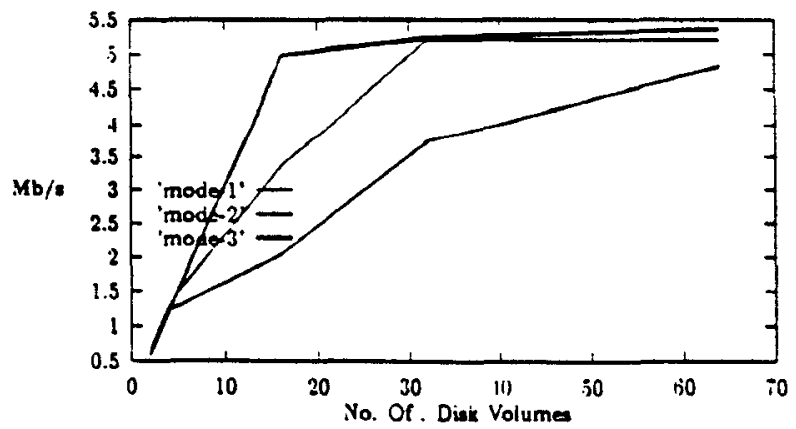

Figure 6: Read for Grid Size $4 * 4$ 


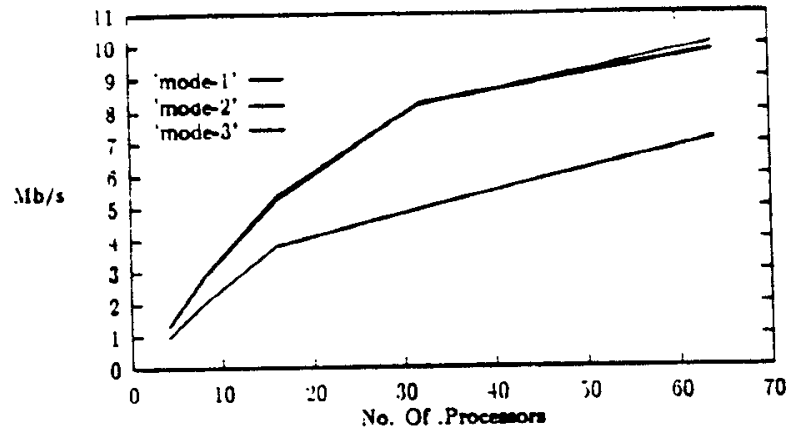

Figure $\mathrm{i}$ : Reading a $16 \mathrm{M}$ File using $4 \mathrm{~K}$ Buffer for 64 Disk Volumes

increases the read th:oughput increases. The highest read throughput was obtained for 64 processors. Modes 1 and 3 perform comparably. The maximum read rate for mode 1 was $10 \mathrm{Mb} / \mathrm{sec}$, while for mode 3 , it was 9.8 $\mathrm{Mb} / \mathrm{sec}$. Due to access ordering and synchronization costs, mode 2 performs worse than the other modes for all cases. As the number of processors increases, the amount of data read per processor decreases. Hence, the individual processors require less time to read. In other words, the available bandwidth at the computational node side increases resulting in an increase in the throughput. However, as the number of processors increases, the rate of increase in the throughput decreases indicating that the bandwidth limitation shifts to the I/O system side.

For studying the performance of each $1 / O$ mode in some more detail, we performed three additional experiments in which the number of processors and the number of disks were varied. For these experiments, a $16 \mathrm{MB}$ file was opened and read by varying number of processors. Figures $8,9,10$ show the effect of varying disk volumes and number of processors for different I/O modes.

Figure 8 shows the performance of the file system for mode 3 . The peak read performance is obtained for 64 disk volumes. This figure also shows that the throughput is proportional to the number of processors. For a 64 processor grid, a $16 \mathrm{MB}$ file was read at 10 $\mathrm{MB} / \mathrm{sec}$. For the same grid size, if the file is stored on 2 disk volumes, the read rate drops to about $900 \mathrm{~KB} / \mathrm{sec}$.

Note that as the number of processor is increased, the knee of the curve is observed at different points for different number of disks. Hence, as indicated earlier, the choice of number of disks depends on how many processors will be involved in an access. More experiments need to be performed to relate this performance to different fle sizes. Note that the knee of the curve in these experiments signifies a point indicating the bandwidth limitation shift from the computational nodes to the I/O system.

Figure 9 shows the results for the same experiment for mode 2. The graph shows nearly same trends as observed for mode 3 . However, for mode 2 , the peak rate

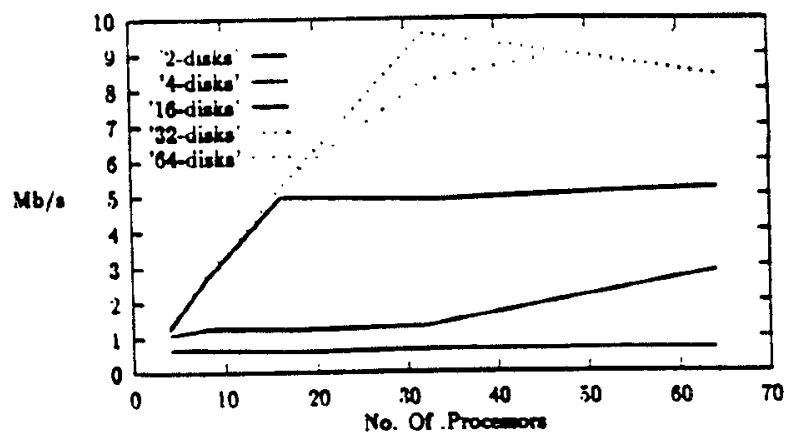

Figure 8: Multicompute Nodes Read (Mode 3 and 16 Mbytes File)

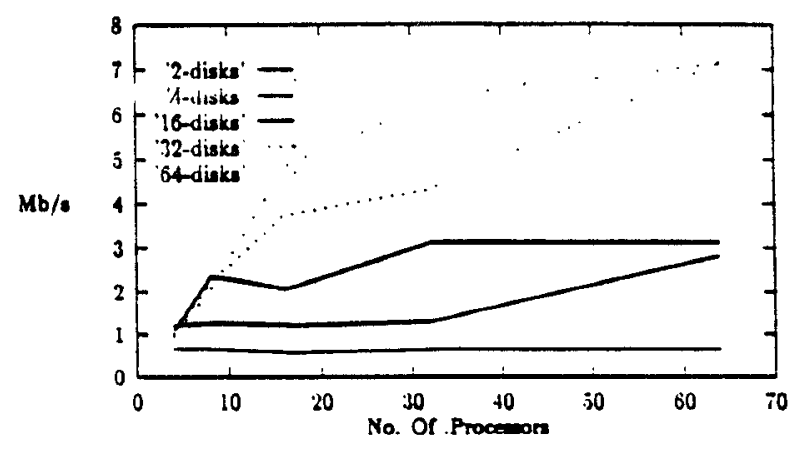

Figure 9: Multicompute Nodes Read (Mode 2 and 16 Mbytes File)

obtained was $7 \mathrm{MB} / \mathrm{sec}$ for 64 processor grid. Note that the performance in mode 2 is sensitive to the order of arrival of the requests because requests must be served in a fixed order. Therefore, we observe a less smooth curve as compared to that for mode 3 .

Figure 10 shows the performance of the multicompute nodes for the mode 1 . The peak throughput is 10 $\mathrm{MB} / \mathrm{sec}$ and lowest observed throughput is $900 \mathrm{~KB} / \mathrm{sec}$. Note that mode 1 serves requests in the order of arrival and does not require synchronization for each processor to finish before going to the next phase. Therefore, it performs slightly better than mode 3 (which requires synchronization). Therefore, mode 1 , is useful for logstructured files or for those computations in which order of accesses does not matter. For example, if the compute nodes perform a search operation in a file, they can access the file in a self-scheduling mode for which mode 1 will provide the best performance.

\section{MODES 1, 2 AND 3: BURST 1/O}

Tables 6 and 7 present a summary of results obtained for reads and writes using burst-mode $I / O$ for various system configurations. The number of processors was varied form $32\left(8^{*} 4 \mathrm{mesh}\right)$ to $512\left(16^{*} 32 \mathrm{mesh}\right)$. Each 


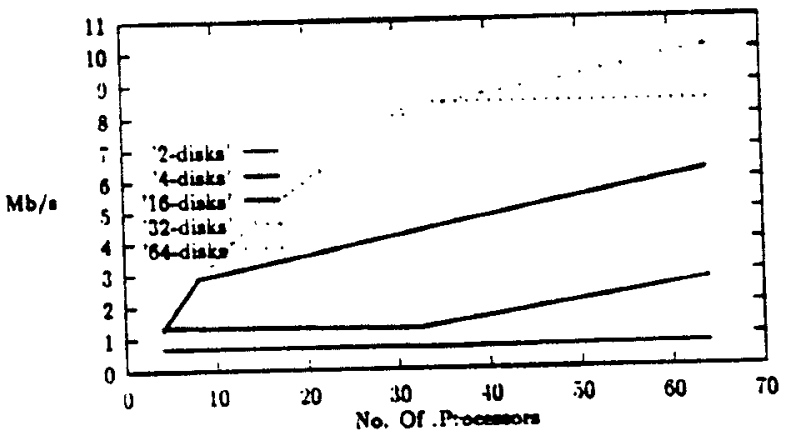

Figure 10: Multicompute Nodes File Reed (Mode 1 and 16 Mbytes File)

Table 6: Read Throughput in Mbytes/sec (Burst Mode)

\begin{tabular}{|c||c||c||c|}
\hline Mesh Size & Mode 1 & Mode 2 & Mode 3 \\
\hline $8^{*} 4$ & $\mathbf{8 . 4 4 7}$ & $\mathbf{8 . 1 5 9}$ & 8.144 \\
\hline $8^{*} 8$ & 4.817 & $\mathbf{8 . 3 1 0}$ & 9.602 \\
\hline $16^{*} 8$ & 8.715 & $\mathbf{8 . 6 8 2 1}$ & $\mathbf{8 . 8 9 1}$ \\
\hline $16^{*} 16$ & 6.519 & 7.18 & 7.169 \\
\hline $16^{*} 32$ & 6.21 & 6.742 & 6.944 \\
\hline
\end{tabular}

processor accessed 1 Mbytes of data, and therefore, the resulting file size varied from 32 Mbytes to 512 Mbytes. Clearly, burst mode $1 / O$ is preferable for large file accesses. Furthermore, a consistent performance is observed over a wide range of processor configurations. However, beyond 256 processors (actually, between 128 and 256) the $1 / O$ system becomes a bottleneck resulting in degraded, but still a comparable performance. It should be observed that read rates were normally 2 to 3 times faster than the write rates.

\section{ARRAY DISTRIBUTION RESULTS}

In large-scale scientific and engineering applications, parallelism is exploited by decomposing the input do main (representing the physical domain model, normally represented by multi-dimensional arrays). However, for load-balancing, expressing locality of access, reducing communications and other optimizations, sev-

Table 7: Write Throughput in Mbytes/sec (Burst Mode)

\begin{tabular}{|c||c||c||c|}
\hline Mesh Size & Mode 1 & Mode 2 & Mode 3 \\
\hline $8^{*} 4$ & 4.19 & 4.310 & 3.217 \\
\hline $8^{*} 8$ & $3.6^{2} 2$ & 4.28 & 3.907 \\
\hline $16^{*} 8$ & 2.60 & 3.1545 & 2.862 \\
\hline $16^{*} 16$ & 2.53 & 2.4255 & 2.479 \\
\hline $16^{*} 32$ & 2.40 & 1.9845 & 2.286 \\
\hline
\end{tabular}

Table 8: Number of $\mathrm{I} / \mathrm{O}$ requests as a function of data distributions

\begin{tabular}{|c||c||c|}
\hline \multicolumn{3}{|c|}{ 1-D Distribution } \\
\hline Distr. Type & No. Of Req. & Data per Req. \\
\hline Column Block & $\mathrm{P}$ & $\frac{N^{2}}{\mathrm{p}}$ \\
\hline Column Cyclic & $\mathrm{N}$ & $N$ \\
\hline Row Block & $N^{*} \mathrm{P}$ & $\frac{N}{P}$ \\
\hline Row-Cyclic & $N^{2}$ & 1 \\
\hline
\end{tabular}

eral decompositions and data alignment strategies can be used

In order to enable a user to specify the decomposition, Fortran D [2], and subsequently High-Performance Fortran [1], have been proposed. The important feature of these extensions is the set of directives that allow a user to decompose, distribute and align arrays in the most appropriate fashion for the underlying computation. The data distribution directives include BLOCK, CYCLIC and BLOCK_CYCLIC distributions (along any dimension of an array).

In this section, we study the performance of the CFS when the processors access files based on the data distributions. Note that the file provides a linear map (e.g. column major) of multidimensional data. Therefore, the number of $1 / O$ requests depend on the specified data distribution on the nodes as shown in Table 8. In this experiment a square character array was distributed across the processors. The array were stored in a column-major form on the disks. The smallest size used was $1^{*} 1 \mathrm{Kbytes}$ and the maximum array that was distributed was $20^{*} 20$ Kbytes ( 400 Mbytes). For each mesh size, the array was distributed in four ways,column block, column cyclic, row block and row cyclic. The following is a summary of experimental results.

Column Block: The column-block distribution implies that the matrix data is distributed along its second dimension onto the processor array. This distribution also conforms with the column-major data distribution over the disk. It requires a single application level $\mathrm{I} / \mathrm{O}$ request per processor and each processor node can read the entire distributed data in one $I / O$ access. The time required to distribute the data column-wise scales with the number of processors for a portion of the configuration space.

Table 9 contains the data for a column-block array distribution. The table shows the size of the array, the number of processors participating in the read, the transaction completion time, and the observed bandwidth. For small size arrays and the number of nodes, the bandwidth of the $\mathrm{I} / \mathrm{O}$ system is underutilized. As the data size and the number of processors increase, the $1 / 0$ bandwidth is more effectively utilized. However, beyond a certain point, the $I / O$ system becomes a bottleneck due to the large number of processors performing $1 / O$, and the need for synchronization.

The read rate increases quickly in proportion to the processor grid size, but plateaued at about 64 proces- 
Table 9: Array Distribution (Column Block) Throughput in MBytes/sec $\left(N_{D}=64\right)$

\begin{tabular}{|c||c|c||c||}
\hline Array & Mesh & Rate & Rate \\
\hline Size & Size & Mode 2 & Mode 3 \\
\hline $1 \mathrm{~K}^{*} 1 \mathrm{~K}$ & 4 & 2.141 & 2.32 \\
\hline $4 \mathrm{~K}^{*} 4 \mathrm{~K}$ & 4 & 4.198 & 7.048 \\
\hline $5 \mathrm{~K}^{*} 5 \mathrm{~K}$ & 16 & 5.098 & 7.44 \\
\hline $4 \mathrm{~K}^{*} 4 \mathrm{~K}$ & 64 & 5.179 & 6.498 \\
\hline $5 \mathrm{~K}^{*} 5 \mathrm{~K}$ & 64 & 6.476 & 7.521 \\
\hline $10 \mathrm{~K} * 10 \mathrm{~K}$ & 256 & 7.038 & 7.65 \\
\hline $20 \mathrm{~K}^{*} 20 \mathrm{~K}$ & 256 & 5.7 & 5.861 \\
\hline $10 \mathrm{~K}^{*} 10 \mathrm{~K}$ & 512 & 5.232 & 5.51 \\
\hline $20 \mathrm{~K}^{*} 20 \mathrm{~K}$ & 512 & 5.517 & 5.63 \\
\hline
\end{tabular}

Table 10: Array Distribution (Column Cyclic) Throughput in Kibytes/sec $\left(N_{D}=64\right)$

\begin{tabular}{|c||c|c||c||}
\hline Array & Mesh & Rate & Rate \\
\hline Size & Size & Mode 2 & Mode 3 \\
\hline $1 \mathrm{~K}^{*} 1 \mathrm{~K}$ & 4 & 160.84 & 229.72 \\
\hline $2 \mathrm{~K}^{*} 2 \mathrm{~K}$ & 16 & 1061.85 & 1527.3 \\
\hline $4 \mathrm{~K}^{*} 4 \mathrm{~K}$ & 16 & 1791.31 & 3057.51 \\
\hline $5 \mathrm{~K}^{*} 5 \mathrm{~K}$ & 64 & 1962.16 & 2191.63 \\
\hline $10 \mathrm{~K}^{*} 10 \mathrm{~K}$ & 256 & 846.92 & 856.43 \\
\hline $20 \mathrm{~K}^{*} 20 \mathrm{~K}$ & 512 & 1522.04 & 1581.15 \\
\hline
\end{tabular}

sors. Degradation in the performance was observed after 256 processors due to a large synchronization overhead. Performance for the small request $\left(1 \mathrm{~K}^{*} 1 \mathrm{~K}\right)$ case was poor.

Column Cyclic: Table 10 shows the read access times for the same parameters but with a column-cyclic data distribution on processors. Even though the degree of parallelism in the data access remains the same, the number of $1 / O$ requests increases (Table 8 ) because each processor must make an individual request for each column. This degrades the access time and the bandwidth as illustrated in Table 10. The degradation in the performance is consistent for all configurations and it ranges between a factor of 2 to 10 as compared to that for column-block distribution.

Table 11: Array Distribution (Row Block) For Modes 2 and $3\left(N_{D}=64\right)$

\begin{tabular}{|c||c||c||c|}
\hline Array Size & Mesh Size & Mode & Rates Kbytes/sec \\
\hline $1 \mathrm{~K}^{*} 1 \mathrm{~K}$ & 4 & 2 & 56.23 \\
\hline $2 \mathrm{~K}^{*} 2 \mathrm{~K}$ & 4 & 2 & 123.84 \\
\hline $4 \mathrm{~K}^{*} 4 \mathrm{~K}$ & 16 & 2 & 114.09 \\
\hline $5 \mathrm{~K}^{*} 5 \mathrm{~K}$ & 16 & 2 & 142.34 \\
\hline $1 \mathrm{~K}^{*} 1 \mathrm{~K}$ & 4 & 3 & 58.64 \\
\hline $2 \mathrm{~K}^{*} 2 \mathrm{~K}$ & 4 & 3 & 154.04 \\
\hline $4 \mathrm{~K}^{*} 4 \mathrm{~K}$ & 16 & 3 & 224.70 \\
\hline $5 \mathrm{~K}^{*} 5 \mathrm{~K}$ & 16 & 3 & 273.11 \\
\hline
\end{tabular}

Row Block: Table 11 shows the performance for reading the data array when distributed in a row-block fashion over the processor array. Since the one-dimensional map of the file on the CFS is in column major order, this read operation essentially requires transposing the data while it is being read from disks to nodes. As shown in Table 8 , the number of logical request is $N^{*} P$. Hence, as observed from Table 11, the performance degradation due to this distribution is almost two orders of magnitude when compared to the performance of the column-block distribution. We do not present performance figures for larger configurations (i.e. large array and system sizes) since the time it took to complete these experiments exceeded practical limits. Thus, we merely conclude that performance for this distribution was at least more than two orders of magnitude worse than the first two configurations. The peak bandwidth obtained was $0.69 \mathrm{Mbytes} / \mathrm{sec}$. This is only $30 \%$ of the slowest case (the $1^{*} 1$ Kbytes case) for the column-block decomposition of Table 9 above. Further, the $1 \mathrm{~K}^{*} 1 \mathrm{~K}$ case for this distribution is 39 times slower than for the equivalent column-block case.

Row Cyclic: The row-cyclic distribution involved the largest number of $1 / O$ requests. Also the request size was the smallest. It took approximately 15 minutes to distribute $1 \mathrm{~K}^{*} 1 \mathrm{~K}$ character array in row-cyclic order versus the $467 \mathrm{msec}$ it would require in the columnblock form. This shows that the direct row distribution of an array is very slow, hence, not possible in practice.

\section{CONCLUSIONS}

In this paper we presented an experimental performance evaluation of the Intel Touchstone Delta CFS. To summerize we conclude,

- The "declustering" of the files improves the read and write performance of the file system for both single and multiple compute nodes.

- For single compute nodes, using the paged I/O mode, the read rate is higher than the write rate. The file access rates depend on the buffer size used in file access. For the file read, normally, as the buffer size increases, the performance improves to a certain point. The buffer size which provides a reasonably good performance for various configurations is $4 \mathrm{~K}$ bytes which is same as the block size and the stripe size. Currently, user has no control over the block size and the stripe size. Further experiments are needed to study the effect of stripe sizes.

- Using the burst-mode I/O, the file access rates improve significantly. It is observed that in general the buffer size for burst $1 / O$ access should be as large as possible for the best performance.

- For the single compute node case, the position of the node in the Touchstone Delta mesh does not affect the file access times. This shows that the "inter-node" hops between the compute nodes are very small relative to the $\mathrm{I} / \mathrm{O}$ performance. This 
shows that there is no possible overhead in using the communication network for the $1 / O$ transactions.

- For the multiple nodes case, the data accessed depends on the file access modes. Mode 0 should be used for reading the shared data, whereas modes 1 to 3 should be used for data distributions. The access throughput depends on the number of processors and it also depends on the total number of disks on which the data is stored. The performance increases initially as the number of processors increase then it remains steady. In general, for small processor grids, the bandwidth is limited on the computational node size, but as the number of processors is increased, it shifts to the I/O system. The point at which this shift occurs depends on the number of processors as well as on the number of disks.

- For the multinode configuration, the performance can be further improved by using the burst mode of operation. Using a large buffer size (2 MBytes) for mode 0 , the peak performance of 23.83 MBytes/sec is observed. For the same mode, the peak write rate is about $7 \mathrm{MBytes} / \mathrm{sec}$. For the remaining three file access mode, burst mode gives a better performance than the paged I/O mode.

- The choice between various modes (except mode 0) depends on the type of access pattern and data organization. Mode 2 should be used only when the size of data access can vary and cannot be determined in advance. Mode 3 should be used if access size is known in advance. Mode 1 should be the choice when order of access is not important because mode 1 does not improve global synchronization.

- Finally it is observed that various data distributions on compute nodes obtain a wide-range of performance from the I/O system. The difference in performance can vary by second orders of magnitude. The distribution most conforming to the distributions on the disks performs the best.

Although, we performed many more experiments, only a selected few are presented in this paper due to space limitations. Many more experiments need to be performed for an in-depth study of each controllable input parameter. However, we believe that these results will be useful for the users of large-scale systems in selecting appropriate I/O scheme for their applications

\section{ACKNOWLEDGMENTS}

We thank Center for Research in Parallel Computing and Concurrent Supercomputing Consortium for access to the Touchstone Delta. We also thank anonymous referees for their helpful comments and suggestions.

\section{REFERENCES}

[1] Forum, H. P. F. High Performance Fortran Language Specification Version 1.0. Tech. Rep. CRPCTR92225, Center for Research in Parallel Computing, Rice University, January 1993.

[2] Fox, G., Hiranandani, S., Kennedy, K., KreMER, U., AND WEN TsENG, C. Fortran D Language Specification. Tech. Rep. Rice COMP TR90-141, Rice University, Houston, Texas, December 1990.

[3] French, J. C., Pratt, T. W., and Das, M. Performance Measurement of the Concurrent File System of the Intel iPSC/2 Hypercube. Journal of Parallel and Distributed Computing (January/February 1993).

[4] Paragon XP/S product overview. Intel Corporation, 1991.

[5] INTEL. Touchstone Delta System Description. Tech. Rep. Intel Advanced Information, Intel Corporation, 1991.

[6] Lionel M. Ni and Philip K. McKinley. A Survey of Wormhole Routing Techniques in Direct Networks. IEEE Computer (February 1993).

[7] M. Barnett and R. Littlefield and D. G. PyNe AND R. VAN DE GEIJN. Global Combine on Mesh Architectures with Wormhole Routing. Proceedings of 7'th International Parallel Processing Symposium (April 1993).

[8] NCUBE CoRPoration. nCUBE 2 supercomputers: Technical overview. Brochure, 1990.

[9] Nitzberg, B. Performance of the iPSC/860 Concurrent File System. Tech. Rep. RND-92-020, NAS Systems Division, NASA Ames, December 1992.

[10] PIERce, P. A Concurrent File System for a Highly Parallel Mass Storage System. In Fourth Conference on Hypercube Concurrent Computers and Applications (1989), pp. 155-160.

[11] RIK Littlefreld. Tuning Communication. Proceedings of the Delta Advanced User Training Class Notes (July 1992), 99-120.

[12] The Connection Machine CM-5 Technical Summary, October 1991. 